\title{
V. Coppia gemellare DZ con Atrofia dell'Iride concordante e Buftalmo discordante
}

\section{Prof. Luigi Gedda e Dott. Sandra Bérard-Magistretti}

\section{INTRODUZIONE}

Vi è motivo di discutere la natura delle malattie primitive dell'iride perchè la dimostrazione dell'eziologia ereditaria di un settore è importante sia dal punto di vista dottrinario sia dal punto di vista pratico, cioè eugenico, ma anche per il fatto che lo studio di una malattia nell'ambito della consanguineità permette di coglierne particolari sintomi, aspetti, stadi ed equivalenti che uno studio limitato al soggetto, che presenta una forma dichiarata, non permetterebbe di evidenziare.

Perciò abbiamo pensato di portare il nostro contributo al problema della malattia ereditaria dell'aplasia iridea. Faremo questo riassumendo la letteratura che si riferisce alla genetica della malattia e poi descrivendo un caso gemellare occorsoci che riteniamo essere, non solo esemplificativo, ma anche, sotto qualche aspetto, dimostrativo, mentre ci riserviamo di tornare con altra casistica clinica sull'argomento.

Gli studi gemellari, che hanno sempre riscosso particolare attenzione da parte dei cultori della specialità oculistica, si sono sviluppati indipendentemente sia nella direzione delle malformazioni dell'iride sia nella direzione dell'idroftalmo, producendo un'interessante casistica che più oltre ricorderemo.

Una recente osservazione gemellare infatti ci ha fornito l'occasione di raccogliere questa bibliografia, ma anche di formulare un'ipotesi che consideriamo interessante e che rappresenta il fulcro del presente lavoro: se cioè non vi sia fondato motivo di ridurre ad unità, almeno in certi casi, lo studio genetistico di queste due sindromi oftalmologiche: l'atrofia dell'iride e l'idroftalmo. 
Infatti la coppia gemellare che ci apprestiamo a descrivere presentava la malformazione iridea in entrambi i gemelli e l'idroftalmo (o buftalmo) monoculare in uno solo dei due gemelli.

\section{LETTERATURA}

\section{a) Generalità}

All'aggiornamento intorno alla letteratura gemellare specifica riteniamo opportuno di premettere una visione d'insieme dei problemi oftalmologici che hanno riferimento con l'atrofia dell'iride per dare occasione ad una rassegna bibliografica soddisfacente anche in ordine ad altri lavori che pubblicheremo sull'argomento.

Fra le alterazioni che possiamo riscontrare a carico dell'iride spicca l'atrofia che può essere primitiva o secondaria e interessare la membrana sia nel suo foglietto mesodermico che nella sua interezza, variando di sede (pars ciliaris o pars pupillaris) come di intensità, da una semplice ipoplasia, magari circoscritta, ad una grave forma di atrofia con scomparsa del foglietto mesodermico ed in seguito con lacerazioni e distruzione anche del foglietto pigmentato.

Le alterazioni a carico dell'iride, inoltre, possono interessare solamente la membrana od anche le formazioni viciniori, cioè l'angolo camerulare, la Desccmet, lo sperone sclerale e la cornea anteriormente, la cristalloide anteriore ed il cristallino posteriormente.

Prescindendo dalle atrofie secondarie (da glaucoma cronico, da tabe, da flogosi, da senilità), l'eziologia delle quali va riferita a quella del processo primitivo, notiamo che le atrofie primitive sono inquadrate sotto nomi diversi come i seguenti: ipoplasia, displasia o aplasia iridea, atrofia congenita anteriore dell'iride, atrofia essenziale congenita, atrofia essenziale progressiva, iridoschisis o atrofia fibrillare senile progressiva, disgenesis mesodermalis iridis et corneae di Rieger. Alterazioni a carico dell'iride si ritrovano pure frequentemente nel capitolo della patologia della camera anteriore inquadrato da Streiff sotto il titolo di displasia marginale posteriore della cornea nel quadro delle malformazioni irido-corneali, nel quale egli riuni e unificò le varie terminologie: embriotoxon corneae posterius (Axenfeld I920), malformazioni del limbo (Saba, 1927), doppio arco senile (Velhagen, I930), anello periferico della Descemet ispessito (Biozzi e Lugli, I935), stria anulare del bordo della Descemet (Karbacher, I940), riunendovi pure la sindrome di Peters e la sindrome di Rieger.

Vario è il grado di interessamento della membrana iridea: da un semplice filamento dell'iride natante nell'acqueo, residuo della membrana fetale di Wachendorf, a depositi pigmentati sulla cristalloide anteriore, da una ipoplasia dell'iride fino all'atrofia, a una corectopia, una policoria, un ectropion dell'uvea, un coloboma. Anche un'aniridia può essere associata, come anche un'eterocromia semplice ed infine un'ipoplasia iridea con alterazione dell'angolo e idroftalmo. 
L'ipoplasia dell'iride può presentarsi sotto vari aspetti: con la membrana di spessore diminuito c questo si vede molto chiaramente nei casi di eterocromia dove un occhio è marrone e l'altro è celeste con un numero inferiore di cripte nelle sue tre porzioni, ciò̀ possono essere conservate nella pars media o nella pars pupillaris o anche nella pars ciliaris. Possono essere conservate le cripte ma il tessuto che le ricopre essere gelatinoso diafano e lasciar trasparire nettamente il foglietto pigmentato sottostante. Questo aspetto dell'iride è stato definito «muschioso». Il colore può essere splendente o nei gradi più avanzati sbiadito, variando dalla normale apparenza cromatica dell'iride fino al colore del foglietto pigmentato sottostante. In un secondo stadio l'iride si presenta appiattita, poche le travature e il disegno è radiale. In seguito l'iride non ha più un colore omogeneo ma si divide in due zone: una parte pupillare bianco-giallastra per il trasparire del muscolo costrittore della pupilla ed una parte molto scura, che occupa la pars media e ciliaris, per il trasparire del foglietto pigmentato; tale aspetto è caratteristico. In seguito, proseguendo l'atrofia, il foglietto posteriore non riesce più a mantenersi integro e comincia a perforarsi con buchi e fessure che possono simulare una policoria. L'orletto pigmentato della pupilla si ectropionizza provocando una midriasi. La pupilla diviene rigida non più reagente anche per l'atrofia del muscolo costrittore. Il colore dell'atrofia iridea nel suo grado estremo generalmente viene definito marron-scuro. Però non tutti i pazienti lo presentano, infatti molti hanno un colorito grigio-piombo assolutamente caratteristico. La causa di questa differenza di colore non è chiaramente identificabile avendo, in altro nostro materiale, osservazioni dei due tipi nella medesima fratria. L'angolo camerulare può presentarsi ampio con tutte le sue strutture normali e bene evidenti o stretto a becco di flauto; si possono riscontrare numerosi granuli di pigmento che vanno ad infarcire le vie di deflusso ed anche goniosinechie nei casi complicati a glaucoma. Si possono trovare finissime listerelle di tessuto irideo che vanno ad inserirsi in posizioni varie dell'angolo ed anche della cornea come autentiche sinechie anteriori. Questi filamenti iridei possono essere chiarissimi, quasi trasparenti, ialini, oppure del medesimo colorito del tessuto irideo e quindi anche scuri o del medesimo colore dei granuli di pigmento che infarciscono l'angolo. Quando si vanno ad inserire direttamente alla cornea, nel punto di contatto con questa, generalmente si nota una piccola opacità della membrana. Possono partire da un tessuto sano, cioè l'iride in quel punto può essere ancora ben conservata nei suoi due strati oppure soltanto nel foglietto pigmentato e la sinechia anteriore corrispondere alla piccola zona mesodermica assente. Talvolta in vicinanza alla sinechia anteriore si nota un foro a tutto spessore, o meglio l'iride è aderente alla cornea ed è lacerata nel suo margine libero. L'aderenza delle due membrane può essere anche molto ampia ed in questi casi l'esame gonioscopico mette in evidenza i corpi ciliari con un aspetto che si riscontra nell'aniridia. Il cristallino può essere indenne ma generalmente mostra delle fini opacità che possono variare da puntiformi, a gocce di cera ed anche come sede, potendosi riscontrare un'opacità centrale nei casi di sindrome di Peters (displasia marginale posteriore di III grado). Generalmente sulla cristalloide anteriore sono evidenti depositi stellari pigmentati, di natura congenita, 
variamente raggruppati e che possono ricoprire completamente la zona pupillare. In altri casi noi possiamo trovare l'angolo ripieno di un tessuto mesodermico indifferenziato che blocca le vie di deflusso e che può provocare un idroftalmo, ma che si può ritrovare anche in fratelli di individui idroftalmici, con condizioni funzionalmente normali. Le condizioni della membrana iridea possono essere stazionarie per tutta la vita o progressive; possono essere ben tollerate dall'occhio, o portare a condizioni gravi fino alla perdita della visione per complicanze intervenute. La complicanza principale è l'ipertensione che generalmente viene considerata secondaria, legata alle condizioni dell'angolo e delle vie di deflusso. Questa ipertensione può svilupparsi in qualsiasi epoca della vita, da quella fetale che porta all'idroftalmo congenito $\mathrm{e}$ in alcuni casi all'idroftalmo maligno quando la C.A. sia assente ed il cristallino e l'iride aderenti alla cornea, a quella senile o pre-senile con l'instaurarsi di un glaucoma cronico che può evolvere fino alla cecità.

Pur essendo l'ipertensione ormai da tutti considerata secondaria, il meccanismo di insorgenza non è del tutto noto. Questo meccanismo consta di due fattori; il primo è la conformazione dell'angolo che può essere anomala, il secondo riguarda il prodotto di disfacimento dell'iride che va ad ingorgare le vie di deflusso magari già alterate in partenza. Resta da risolvere la variabilità del momento di insorgenza pur essendo apparentemente uguali in certi casi le condizioni obiettive. Anche in un medesimo individuo i due occhi non si comportano talvolta nella medesima maniera. Generalmente l'alterazione iridea è bilaterale, ma quasi mai allo stesso stadio. Talvolta succede che l'occhio il quale ha l'iride meglio conservata e l'angolo camerulare più libero sia quello nel quale il glaucoma è più progredito. Un altro punto da prendere in considerazione è il comportamento di questi glaucomi: generalmente non raggiungono una tensione molto elevata, danno facilmente edema corneale anche fugace con ipertensioni modicissime e frequentemente, una volta divenuti assoluti, presentano una cheratite bollosa che dà generalmente pochissimo fastidio al paziente. Queste alterazioni parlano di una compartecipazione della cornea anche se non sappiamo come, e vanno ricordate perchè noi spesso abbiamo trovato in questi pazienti distrofie epiteliali ed endoteliali, queste ultime riscontrate anche da Chandler. Sono pure state riscontrate alterazioni a carico di altri organi ed apparati: emangiomi, dermoidi, alterazioni ossee, alterazioni a carico dei denti, colobomi che possono interessare l'iride fino al cristallino, alla coroide. Il coloboma a carico dell'iride può essere in qualsiasi sede ma generalmente è inferiore, nella sede, cioè, dove avviene la chiusura della fessura fetale. Può essere a tutto spessore o interessare soltanto il foglietto anteriore, oppure presentarsi nel medesimo individuo in un occhio a carico di un solo foglietto e nell'altro a carico di tutti e due; varie ipotesi furono prospettate sulla possibile etiologia. Streiff, Poliakowa-Spector ritengono che l'ipoplasia sia un'anomalia congenita consistente in un'abnorme regressione dello strato mesodermico, dal quale si forma lo stroma irideo e la pupilla, regressione che non si fermerebbe con la nascita. Su quest'ipotesi venne fissato da molti il punto di partenza dell'atrofia iridea. Bitter infatti osservò tre casi in cui essa partiva dal piccolo cerchio. Rübel pensa che vi sia stato un arresto di sviluppo, Kreiker ritiene 
che i processi citolitici che nella vita embrionale provocano il riassorbimento della membrana pupillare, non si arrestino con la nascita, ma rimanendo attivi, interessino poi il tessuto irideo normale, Feingold pensa con Rübel ad un disturbo circolatorio del piccolo cerchio dell'iride che provochi in un secondo tempo l'alterazione del tessuto. Lane e Zentmayer ipotizzano una sclerosi vasale, mentre altri pensano ad una eredo-degenerazione mesodermale che rientrerebbe nel capitolo delle distrofie abiotrofiche. Kamenestzky ritiene che nell'ammalato colpito da alterazioni di questo tipo vi sia una predisposizione ereditaria; l'atrofia verrebbe ad evidenziarsi solo verso i Io-I2 anni e i motivi causali sarebbero disfunzioni ghiandolari (anche in queste sarebbe presente un meccanismo ereditario). Un'altra ipotesi riconduce l'ipoplasia dell'iride ad alterazioni del simpatico dato che la sindrome di Horner può essere associata ad eterocromia e l'iride appare anche ipoplasica oltre che alterata quanto a colore. Le lacune del foglietto pigmentato non sarebbero mai primitive ma secondarie allo stiramento delle fibre muscolari che provocherebbero la lacerazione del foglietto ectodermico. Questi non avendo la parte elastica superficiale, rimane indebolito e facilmente si rompe dando luogo alle caratteristiche fessurazioni.

\section{b) Bibliografia genetistica riguardante 1'Atrofia dell'Iride}

Kamenestzky descrive nel 1925 una famiglia in Siberia, nella quale la quasi totalità degli individui era colpita da atrofia del foglietto anteriore dell'iride in entrambi gli occhi, con glaucoma secondario.

Pellaty nel 1926 riporta un caso familiare di atrofia iridea che in alcuni membri della famiglia era appena accennata, essendovi soltanto un appiattimento dell'iride e pupilla midriatica.

Rossano nel i 934 descrive due casi di ipoplasia del foglietto mesodermico dell'iride, che in uno dei due pazienti era complicata da glaucoma e riduzione visiva; i due malati appartenevano a due generazioni.

RIEGER nel i935 descrive la sindrome familiare «disgenesis mesodermalis iridis et corneae ", che si trovava associata ad un'anodontia parziale vera.

THEODORE nel 1944 descrive la displasia marginale in due sorelle.

Resehrt e Mikja nel i952 riportano una famiglia nella quale vi erano 3 casi di disgenesis mesodermalis associato ad anodontia (in 3 generazioni).

François e De Rouck nel I953 riportano un albero genealogico in cui vi era aplasia congenita bilaterale e stazionaria dell'iride che interessava inoltre ambedue i foglietti, mesodermico e ectodermico, nella loro porzione circumpapillare. Da quanto osservato, gli AA. propendono per una forma incompleta di aniridia e sono dell'opinione che un medesimo gene abbia trasmesso le anomalie.

Cornalba nel 1954 descrive 3 casi di atrofia primaria del foglietto anteriore dell'iride presente nella stessa famiglia; vi erano disfunzioni endocrine e disturbi psico-neurotici che l'A. ritiene possano aver influito su un terreno predisposto ereditariamente. 
KitTeL nel 1956 riporta un caso familiare comprendente nonno, padre e figlio di 4 anni: il bambino era oligofrenico e aveva una parziale anodontia; non c'erano aderenze dell'iride all'anello di Schwalbe; in tutti e 3 vi era inoltre una mascella ipoplasica.

Rossetti e Betetto nel ${ }_{9} 956$ descrivono una famiglia con un membro che presentava ipoplasia dell'iride, un altro con embriotoxon e residui mesodermici, un terzo membro con alterazioni tipo von Hippel al fondo dell'occhio.

UNGER nel 1956 cita due casi familiari di disgenesis mesodermalis di Rieger presenti in madre e figlia; l'A. imputa la genesi di questa malformazione all'ectoderma dato che l'anodontia parziale vera ha questa origine.

\section{c) Bibliografia genetistica riguardante 1'Idroftalmo}

Johnson ( 1898 ), sotto il titolo « Buphthalmia: an interesting series of cases occurring in the same family ", riporta il caso di tre fanciulli affetti da idroftalmo in una fratria di 6 il cui padre è monocolo in seguito ad un'affezione indeterminata; la cecità è stata constatata all'età di 7 anni. Esaminato nell'età adulta, il padre presenta l'OD normale, mentre l'OS ha un visus spento, per cataratta complicata.

Bondi (ig03), nel lavoro "Megalophthalmus und Hydrophthalmus in einer Familie», segnala nella stessa famiglia madre ed un figlio affetti da megalocornea, due figli morti in tenera età con grossi occhi ed infine l'ultimo nato affetto da idroftalmo.

Wallenberg (I9Io) riporta un'osservazione «Erbliches, juveniles Glauckom in vier Generationen » in cui l'affezione si è presentata all'età di 20 anni. WESTERLund (1947) ha già fatto osservare che questo caso non poteva essere un idroftalmo ma un glaucoma giovanile.

TAKAGI e ODA (I934) riportano un albero genealogico di 6 generazioni nel quale è possibile riferire due soggetti di sesso maschile e 6 di sesso femminile colpiti da idroftalmo.

Brons (1937) ha studiato 7 famiglie la cui genealogia risale molto in alto nel tempo e vi ha trovato numerosi casi di idroftalmo. Ha reperito inoltre un matrimonio consanguineo in 3 famiglie, ma uno di questi risaliva all'undicesima generazione.

BaratTa nel 1937 osservò due casi, madre e figlio, nei quali oltre all'idroftalmo vi era corectopia policoria e cataratta.

Cresseri e Becherle (I944-45), studiando un albero genealogico risalente all'anno i6oo, trovano 6 fratrie con I I soggetti colpiti su 32, il che corrisponde ad una penetranza vicina al $100 \%$.

DALSGARD-NIELSEN (1945) riportano 2 casi ereditari di idroftalmo su una statistica di 40 casi.

Westerlund (1947) trova in tutto il suo materiale i 8 casi di idroftalmo riguardante ${ }_{0} 5$ fratrie, in cui il numero totale dei membri è di 462 . Vi è dunque una frequenza degli individui colpiti del $25,5 \%$. 
Falls nel 1949 trovò in una famiglia idroftalmo, microcornea, embriotoxon, policpria, corectopia ed ectropion uveae. KLuiskens (1950) segnala due casi nei quali l'idroftalmo si ritrova in tre generazioni.

Gallenga e Matteucci nella relazione sull'idroftalmo del $195^{2}$ descrivono un caso familiare nel quale su una fratria di 6 individui, 2 avevano idroftalmo e tutti numerose anomalie congenite, quali nistagmo, atrofia dell'iride, discromatopsia e cataratta congenita; l'esame gonioscopico mostrò in 4 di essi alterazioni variabili dell'angolo, da un quadro quasi normale a un grande sviluppo dei processi iridei; nei 2 fratelli idroftalmici vi era infine un'inserzione alta dell'iride con abbondante tessuto indifferenziato; secondo gli AA. l'atrofia del foglietto anteriore dell'iride alla base, sarebbe un reperto caratteristico dell'idroftalmo. Nel capitolo poi sull'idroftalmo maligno gli AA. pongono l'alterazione consistente nell'opacità centrale della cornea con sinechia anteriore dell'iride, presenza della membrana pupillare e G.A. assente.

Gianferrari, Cresseri e Maltarello (i954), studiando 9 famiglie in cui l'affezione è presente in molte generazioni, trovano I 6 soggetti affetti su 49, il che corrisponde ad una penetranza vicina al $100 \%$.

Delmarcelle ( I957) riporta 2 casi: I caso, di idroftalmo bilaterale apparso all'età di 4 mesi in un fanciullo il cui padre e la zia paterna sono affetti da idroftalmo congenito bilaterale di forma grave. Non si rivela consanguineità nella famiglia, ma l'albero genealogico non potè essere risalito molto in alto nall'ascendenza.

II caso, di idroftalmo bilaterale in un fanciullo di 5 mesi. Il padre è affetto da idroftalmo bilaterale. Un primo figlio, dell'età di 2 anni, è sano. La madre, ugualmente indenne dall'affezione oculare, ha avuto, da un primo matrimonio, 3 figli sani. Nessuna consanguineità dichiarata nella famiglia, salvo che dal lato materno in cui i nonni sono cugini.

WeEkers S. e R. hanno illustrato una famiglia che presentava, oltre all'idroftalmo, megalocornea, cmbriotoxon, anomalie riguardanti l'angolo irido-corneale; studiano anche la struttura dell'iride, forma e posizione della pupilla.

Passiamo ora brevemente in rassegna i casi gemellari:

Kienle e Pugmine (1934) descrivono una coppia MZ di a. 6 nella quale l'idroftalmo era monolaterale e speculare. Non era concordante invece la gravità. Il bulbo aveva incominciato ad ingrandirsi all'età di 3 mesi. L'anamnesi familiare era silente. Le condizioni obiettive erano le seguenti: OS: Visus $1 / 1$, tono $65 \mathrm{~mm} . \mathrm{Hg}$., Fundus: escavazione profonda della papilla. Alla L.a.F. si notavano numerose rotture della Descemet ed un'iride atrofica. L'OD era indenne. Il cogemello presentava all'OD bulbo molto ingrandito, cornea opaca, iride di aspetto muffo ed il cristallino ridotto ad una massa biancastra sporgente dall'iride, fondo inesplorabile. L'OS era indenne. In ambedue i gemelli l'indagine radiologica mostrava un allargamento dell'orbita contenente l'occhio idroftalmico.

Poтts (1950) riferisce pure lui un'osservazione gemellare monozigotica nella quale l'idroftalmo era monolaterale e speculare. L'anamnesi familiare era silente. La ma- 
lattia datava dalla nascita. All'esame obiettivo presentavano: I gemello OD: Visus spento, bulbo ingrandito in toto, iride sottile, stirata, con poche cripte e residui della membrana pupillare; al fondo, papilla escavata ed atrofica. OS indenne. II gemello OS: il reperto era uguale al cogemello, solo vi erano in più rotture della Descemet e cataratta complicata; OD indenne. L'A., eseguito un reperto istologico, ha trovato assenza del canale di Schlemm, lo sperone sclerale ed il trabecolato corneosclerale rudimentali. Iride atrofica.

Anderson (1939) riporta due casi. Il primo si riferisce ad un'osservazione di GaUlT (1937) consistente in idroftalmo bilaterale concordante in gemelli MZ. Essi erano stati operati con esito infausto: i pazienti erano ciechi.

Il secondo caso si riferisce ad un'osservazione di Duncan e consisteva in una coppia probabilmente monozigotica che presentava un idroftalmo bilaterale concordante e contemporaneamente distacco di retina.

Castelli (1940) descrive una coppia gemellare bisesso di $3 \mathrm{~m}$. nata prematura al settimo mese, che presentava in maniera discordante l'idroftalmo. Al reperto autoptico di uno dei membri deceduto per eresipela trovò assenza del canale di Schlemm e atrofia dell'iride. L'idroftalmo era congenito.

Massimeo (i950) descrive una coppia maschile MZ di 6 a. con idroftalmo monolaterale concordante. Anamnesi familiare silente. Il reperto era identico e consisteva in bulbi ingranditi, cornea aumentata nei suoi diametri principali, appiattita, con rotture della Descemet. Camera anteriore profonda ed iride atrofica nella pars ciliaris. Residui della membrana pupillare di Wachendorf.

\section{DESGRIZIONE DELLA COPPIA GEMELLARE DZ NADIA E GLAUDIO Or. DI 20 MESI}

NADIA, I nata (cfr. fig. I)

\section{Anamnesi}

Nata da gravidanza gemellare decorsa normalmente. Parto eutocico, peso alla nascita $\mathrm{Kg} .2,900$; prima suzione valida nelle 24 ore. Cordone ombelicale caduto in quarta giornata senza residua secrezione. Allattamento materno esclusivo per 15 giorni, poi misto con latte in polvere mezza crema. Prima minestrina a 6 mesi. Primo dente a dieci mesi. Primi passi a 14 mesi. Non ha subito le vaccinazioni antivaiolose. Durante l'allattamento artificiale le sono stati somministrati fattori vitaminici A e D e vitamine del gruppo B e G.

Alla fine del quarto mese di vita aumento delle evacuazioni con emissione di feci acide. Venne fatta diagnosi di dispepsia fermentativa che venne curata con adatta dieta. A dieci mesi di vita fu morsa da un topo di fogna per cui venne ricoverata all'Ospedale di Nettuno e qui sottoposta a sieroprofilassi antitetanica. A I I mesi secondo episodio di dispepsia fermentativa curato con la somministrazione di batteriofago e preparati digestivi. A I4 mesi parotite epidemica guarita senza complicazioni. 


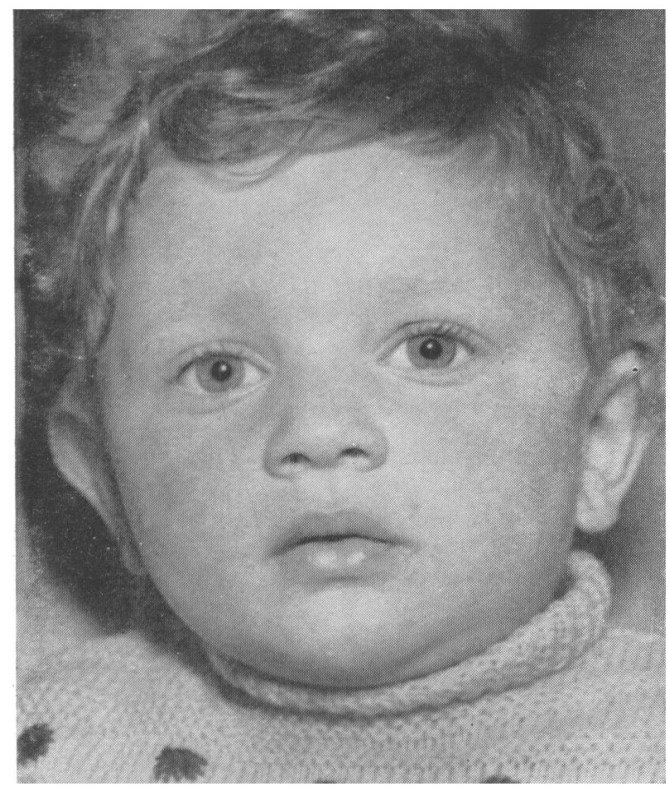

Fig. I

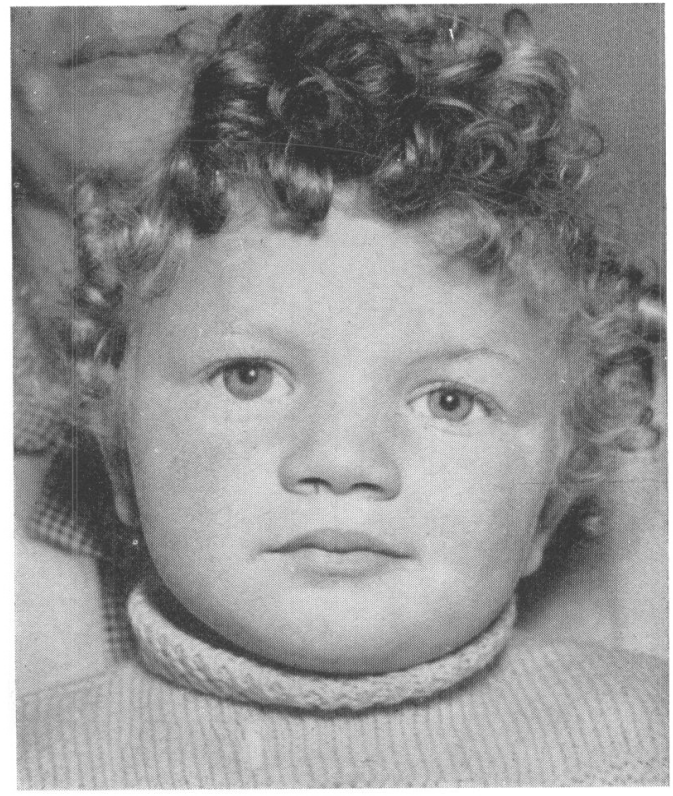

Fig. 2

\section{Esame obiettivo}

Mesi 20, Kg. i I,49o. Condizioni generali buone. Cute rosea; mucose visibili rosee, pannicolo adiposo ben conservato. Turgore conservato. Linfoghiandole di grandezza normale nelle comuni stazioni linfoghiandolari.

Cranio: mesaticefalo; fontanella anteriore chiusa. Negativo l'esame esterno a carico degli orecchi e del naso.

Cavo orale di forma normale. Denti i 3 . Ipertrofia delle tonsille palatine.

Apparato respiratorio: torace leggermente svasato alle basi. Alla percussione suono chiaro polmonare. All'ascoltazione murmure vescicolare a destra, a sinistra presenza di ronchi e sibili.

Apparato cardio-vascolare: aia cardiaca nei limiti. Toni all'ascoltazione netti. Azione cardiaca ritmica.

Addome leggermente globoso, cicatrice ombelicale introflessa. La milza non è palpabile. Il fegato si palpa all'arco costale. Limite superiore della ottusità epatica al $V$ spazio intercostale.

Sistema nervoso: motilità attiva e passiva normale. Riflessi rotulei e achillei normali. Nulla a carico degli organi genitali.

\section{Esame oftalmologico}

OD: Bulbo piuttosto voluminoso con cornea di forma abbastanza grande; C.A. profonda e otticamente vuota; iride ipoplasica nel suo foglietto mesodermico; cristallino e vitreo trasparenti; fondo oculare normale. Tono: $18 \mathrm{~mm}$. $\mathrm{Hg}$. 
OS: Cornea liscia, lucida e trasparente, leggermente meno grande dell'altra. L'iride si presenta meno ipoplasica. Cristallino e vitreo trasparenti; fondo oculare normale. Tono: $18 \mathrm{~mm}$. Hg.

La motilità è normale in tutti i campi di sguardo. Gli annessi sono normali.

Esame immunoematologico: $\mathrm{B}, \mathrm{MN}, \mathrm{P}, \mathrm{CDE} / \mathrm{ce}$.

CLAUDIO, II nato ( $f f r$. fig. 2)

Anamnesi

Nato da gravidanza gemellare decorsa normalmente. Parto fisiologico, peso alla nascita Kg. 2,700. Prima suzione valida nelle 24 ore. Cordone ombelicale caduto in sesta giornata senza residua secrezione. Allattamento materno esclusivo per I 5 giorni, poi misto con latte in polvere mezza crema. Prima minestrina a sei mesi. Primo dente a 12 mesi. Primi passi a I 4 mesi. Non ha subito le vaccinazioni antivaiolose. Durante l'allattamento artificiale gli sono stati somministrati fattori vitaminici A e D e vitamine del gruppo B e G.

Alla fine del quarto mese aumento delle evacuazioni con emissioni di feci acide. La dispepsia fermentativa fu curata con adatta dieta.

All'età di 7 mesi Claudio è stato portato a visita specialistica per un ingrossamento progressivo del bulbo oculare destro che si presentava con la cornea biancastra senza peraltro provocare dolore. All'EO presentava: Tono: $40 \mathrm{~mm}$. Hg. con edema corneale imponente. Idroftalmo. Ricoverato in corsia veniva operato correttamente di ciclodialisi, con esito soddisfacente.

Ail'ottavo mese febbre elevata con disfagia per cui fu fatta diagnosi di tonsillite acuta che venne curata con penicillina in supposte.

A 14 mesi febbre senza segni di localizzazione durata circa 5 giorni.

\section{Esame obiettivo}

Mesi 2o, Kg. I0,670. Condizioni generali: buone. Cute rosea; mucose visibili rosee. Pannicolo adiposo ben conservato. Turgore conservato. Linfoghiandole normali nelle comuni stazioni.

Cranio: mesaticefalo. Fontanella anteriore chiusa. Il globo oculare destro appare più grande di quello di sinistra. Negativo l'esame esterno a carico degli orecchi e del naso.

All'esame del cavo orale si nota una lingua con zone di disepitelizzazione a tipo "lingua a carta geografica ". Le tonsille palatine sono ipertrofiche e $\mathrm{i}$ denti sono i 1 .

\section{Apparato respiratorio}

Torace svasato alle basi con ingrossamento condro-costale bilateralmente. Alla percussione del torace suono chiaro polmonare e alla ascoltazione murmure vescicolare su tutti gli ambiti.

\section{Apparato cardiovascolare e Addome}

Aia cardiaca nei limiti. Toni cardiaci netti all'ascoltazione. Azione cardiaca ritmica.

Addome di forma e volume normale. Cicatrice ombelicale introflessa. La milza non è 
palpabile. Il fegato deborda di un centimetro dal lato costale. Limite superiore dell'ottusità epatica al V spazio intercostale.

\section{Sistema nervoso}

Riflessi rotulei ed achillei normali. Motilità attiva e passiva normali. Nulla a carico degli organi genitali.

\section{Esame oftalmologico}

OD: bulbo ingrandito in toto con cornea grande, liscia, Iucida e trasparente, C.A. ot ticamente vuota e di profondità aumentata. Iride di colorito azzurro-piombo estremamente ipoplasica nel suo foglietto mesodermico, soprattutto nella pars ciliaris. Cristallino e vitreo trasparenti, papilla pallida ed escavata. Tono: $18 \mathrm{~mm}$. $\mathrm{Hg}$.

OS : bulbo più piccolo dell'altro, però sempre piuttosto grande, cornea liscia, lucida e

IPOPLASIA DELL-IRIde

Buftalmo

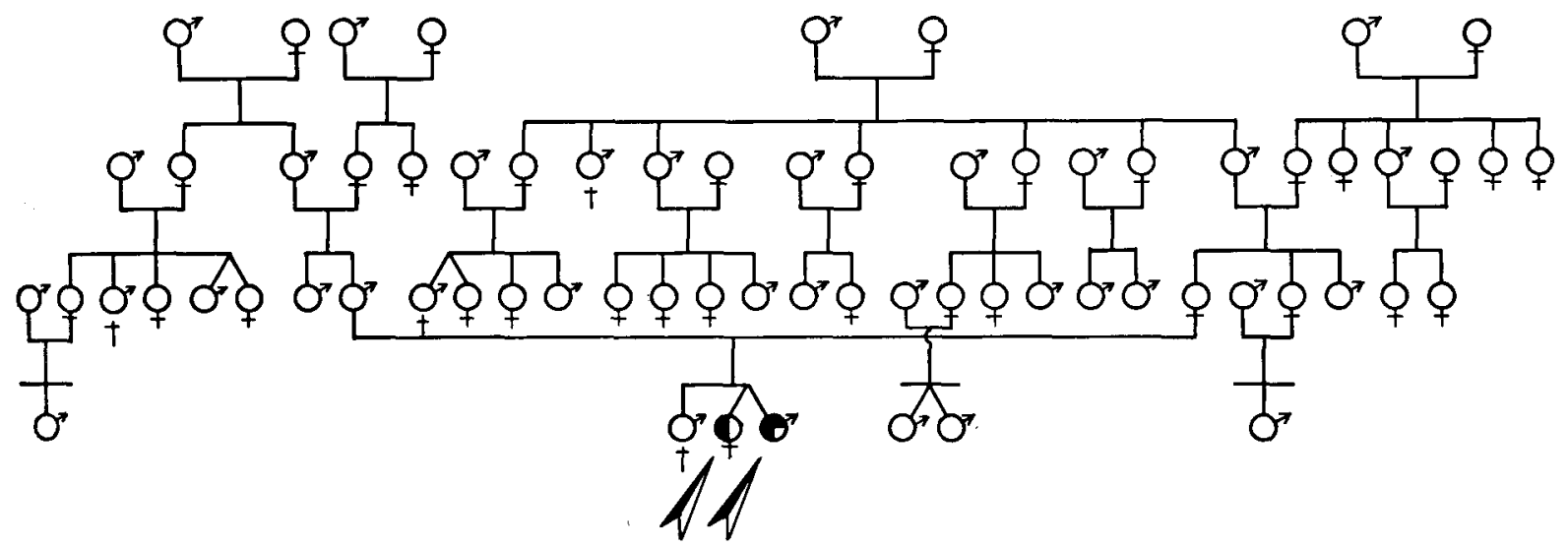

Fig. 3 - Albero genealogico della Famiglia Or.

trasparente, iride ipoplasica nel suo foglietto mesodermico, ma meno dell'altro occhio; cristallino e vitreo trasparenti, fondo normale. Tono $18 \mathrm{~mm}$. $\mathrm{Hg}$.

La motilità è normale in tutti i campi di sguardo. Gli annessi sono normali.

Esame immunoematologico: $\mathrm{O}, \mathrm{MN}, \mathrm{P}$, cde/ce

\section{REPERTO FAMILIARE}

I genitori dei gemelli presentano entrambi iridi di colorito azzurro con stroma irideo normale seppure non molto spesso. Gli occhi non sono molto grandi ed hanno refrazione normale. Non consanguineità e non malattie oculari degne di nota nel gentilizio (cfr. fig. 3). 


\section{DISGUSSIONE}

Incominceremo riferendo un caso sotto alcuni aspetti analogo al nostro ma che non appartiene alla letteratura perchè inedito. Tale caso di atrofia dell'iride e idroftalmo in gemello DZ ci è noto perchè segnalatoci dal Ch.mo Prof. Santonastaso Direttore dell'Istituto di Clinica Oculistica dell'Università di Padova, che sentitamente ringraziamo.

\section{Caso clinico di C. R. di a. 6 da Tritiana (Belluno)}

\section{Anamnesi familiare}

Non consanguineità negli ascendenti.

A.F.P.: nato a termine da parto eutocico, gemellare, dizigotico. Ha 5 fratelli viventi e sani. Dalla nascita $\mathrm{i}$ genitori hanno notato che il bambino vedeva meno degli altri. $\mathrm{Fu}$ visitato a 6 mesi da uno specialista che non prescrisse alcuna terapia. Fu ricoverato a 8 mesi per intervento all'OS.

La cogemella, secondo i genitori, è sana anche dal punto di vista oftalmologico.

Durante la gravidanza la madre ha sofferto di disturbi gastro-intestinali, non malattie a carattere infiammatorio o infettivo; soltanto $20 \mathrm{gg}$. prima del parto ha avuto un'eruzione cutanea con ponfi, afebbrile. Tale gravidanza è avvenuta dopo che il marito è tornato dalla prigionia.

\section{Esame obiettivo}

Regione orbitaria e segmento anteriore OO. Movimenti incoordinati dei bulbi e qualche scossa di nistagmo.

OD: bulbo ingrandito in toto dalla nascita, opacità centrale della cornea a tipo degenerativo, cui aderisce posteriormente un tessuto di natura organizzativa con foro pupillare piuttosto miotico, irregolare; aderente alla massa stessa si intravede il cristallino. Embriotoxon corneae posterius.

OS: il bulbo appare un po' più piccolo del destro. Opacità degenerativa della cornea a tipo bandelletta. Camera anteriore bassa. Atrofia dell'iride. Ampio coloboma chirurgico ore XII occupato dal cristallino opacato.

$O O$ : non si avverte alcuna reazione alla luce.

\section{Giudizio diagnostico}

L'OD idroftalmico (è ingrandito in toto), è affetto pure da un complesso malformativo ed eredo-degenerativo con distrofia epiteliale della cornea, Embriotoxon, residui organizzativi del segmento uveale. Presenta inoltre un cristallino in gran parte opacato. Il visus è spento.

L'OS pure idroftalmico (ma è meno grande dell'OD), presenta inoltre una cataratta congenita e probabili fatti malformativi. Pure in questo occhio il visus è spento. 
Consegnando alla letteratura il caso rilevato a Padova ci sembra logico confermare la diagnosi di idroftalmo bilaterale con fatti malformativi, discordante in gemello maschio di coppia DZ bisesso. Però non siamo in grado di affermare se l'atrofia iridea presente nel gemello idroftalmico fosse o no presente anche nella cogemella, ammaestrati come siamo, dall'analogo caso gemellare che stiamo descrivendo, del fatto che i familiari, mentre danno peso alle variazioni di volume di un globo oculare, di solito non attribuiscono importanza alle anomalie dell'iride quando sono accompagnate da sufficiente visus e tanto meno apprezzano le variazioni che non alterano il forame pupillare in quanto riguardano la struttura dei foglietti che compongono l'iride.

Invece è proprio su questa contestualità dell'atrofia iridea che i nostri due gemelli, benchè dizigotici, presentano, che ci sembra doveroso di imperniare la discussione del caso del gemello Or, in quanto esso suggerisce un'ipotesi interpretativa di qualche interesse.

Noi sappiamo infatti che l'ipoplasia dell'iride nei suoi vari gradi riconosce una base congenita che si instaura durante la vita fetale come un'alterazione di sviluppo che può ripetere a cause diverse, come si è dimostrato nel paragrafo della letteratura, ma anche ad un'eredità morbosa per lo più di tipo dominante.

D'altra parte noi sappiamo anche che l'idroftalmo è presente in un solo membro della coppia e che questa deformazione macroscopica fu rilevata dai familiari solo nel terzo mese dopo la nascita, cioè non appare essere una malformazione congenita.

Mentre dunque i nostri due gemelli presentavano presumibilmente all'atto della nascita l'atrofia dell'iride che anche oggi presentano, solo il maschio, ma a distanza dalla nascita, andò sviluppando il buftalmo per cui giunse alla nostra osservazione. Di qui una possibile interpretazione la quale consisterebbe nel considerare come eredità primaria la malformazione iridea e come complicazione fenotipica dovuta al convergere di altri fattori idiotipici, paraidiotipici e paratipici, l'idroftalmo discordante che abbiamo descritto.

Quest'interpretazione bene si accorda con il tipo dizigotico della gemellanza in esame perchè l'eredità relativamente diversa può aver fatto sì che nella gravità il processo malformativo irideo, fondamentalmente concordante, fosse, quanto all'espressione, diverso, cioè più grave, a carico del maschio, oppure che altre circostanze contestuali discordanti abbiano aggravato il processo morboso a carico del maschio, e non della femmina, mediante la complicazione idroftalmica.

Con la surriferita ipotesi si accorda anche la negatività oftalmologica del reperto familiare in quanto quelle atrofie dell'iride, che i profani di solito non rilevano, possono aver marcato l'eredità del carattere negli ascendenti e nei collaterali senza che l'anamnesi fosse in grado di denunciarle, in assenza della complicanza idroftalmica, dunque non necessaria per stabilire la natura genotipica dell'affezione.

Bisogna però dichiarare che l'interpretazione sulla quale ci siamo soffermati, benchè assai probabile, viene presentata come un'ipotesi la quale ha come alternativa un'altra possibile ipotesi. Può darsi infatti, in via presuntiva, che non di una 
sola eredità si tratti ma di due distinti e coagenti meccanismi ereditari, l'uno relativo all'atrofia dell'iride, l'altro relativo all'idroftalmo, i quali, data la diversità genotipica di gemelli $\mathrm{DZ}$, possono essere diversamente rappresentati nei due membri.

Per decidere quale delle due ipotesi ha maggior ragione di essere adottata, bisogna riferirsi a studi più vasti possibilmente condotti su scala gencalogica, più cospicua di quella offerta da una coppia gemellare dizigotica.

\section{Riassunto}

$\mathrm{Si}$ riferisce intorno ad una coppia gemellare dizigotica bisesso di mesi 7 nella quale i due membri presentano atrofia dell'iride e solo uno, il maschio, buftalmo acquisito intorno al terzo mese. Si formula l'ipotesi che l'eredità primitiva consista nella malformazione iridea e che in questo caso il buftalmo sia secondario a tale malformazione.

\section{Bibliografia}

Abin, G.: Essential atrophy of the iris. Bull. Soc. Ophtal. Egypte, 42, $206,1952$.

Anroguè, E., Wolf, J. A.: Aniridia bilateral con ptosis y cmbryotoxon (Fxamen gonioscópico). Arch. Oftal B. Aires, 23, I4I, I948.

Alaerts, L.: Ies atrophies temporales de l'iris dans les glaucomes chroniques. Bull. Soc. beige: Ophtal, ro7 2 I9, I954.

Alagna, G.: Atrofia girata della coroide e retina (contributo clinico e patogenetico). Arch. Ottalm., 58, 33, I954.

Ammon, V.: Die klinische Darstellung der angeborenen Krankheiten und Bildungsfehler der Augen. Reimer, Bcrlin, 1867 .

Anderson, J. R.: Hydrophtalmia or congenital glaucoma. London, Cambridge University Press, 1939.

Argyll-Robertson: Discussione a CROSS: Hydrophthalmos. Trans. ophtal. Soc. U. K. II, 239 , I89I.

Axenfeld, Th.: Embryotoxon corneae posterior. Ber. dtsch. ophth. Ges. Heidelberg., 42, 301 , 1920.

Baillart, Magitot: Le régime circulatoire du glaucome. Ann. Oculist., Paris, 162,723 , I925.

Baitsch, H.: Ueber Korrelationen und Generalfaktoren in der Struktur und Pigmentierung der menschlichen Iris. Homo 5 , I 954 .

Bailantyne, A. J.: Proc. R. Soc. Med., 26, i3, 1933.

Baratta, O.: Alterazioni congenite familiari dell'iride. Bo'l. Oculist, i6, 339, 1937.

BARKAN, O.: The structure and function of the angle chamber and Schlemm's canal. Arch. Ophthalm., I.5, IOI, I 936 .

BAR'Tels, M.: Ueber Blutgefässe des Auges bei Glaukom und über experimentelles Glaukom durch Versperrung von vorderen Blutbahnen. Z. Augenheilk. I 4, pp. 103, 258, 458, 1905.

Begut, H., Bonnefous, J.: Atrophie essenticlle progressive de l'iris. Bull. Soc. ophtal. France, 591, I955.

-.- - Atrophie essentielle progressive de l'iris. Ann. d'Oculist., Paris, I 89, 331, I956.

Bentzen, C., Leber, 'T.: Ueber die Filtration aus der vorderen Kammer bei normalen und glaukomatösen Augen. Graefes Arch. Ophthal., 4I, 208, 1895.

BF,RG, F.: Acta ophthal., Kbh. Io, 568, ig32.

liercmeister: Zwei Fälle von angeborener Irideremie. Graefes Arch. Ophthal., 59, ior, igo4.

Bietti, G. B.: Atrofia dell'iride con fenestratura di inusitata ampiczza in occhio idroftalmico. Boll. Oculist., I2, I 72,1933 . 
Biozzi, G., Lugli, L.: Ringförmige, periphere Verdichtung in der Gegend der Descemet (Ein neuer Spaltlampenbefund). Gracfes Arch. Ophthal., 134, 287, 1935.

Bistis, J.: Contribution expérimentale sur le rôle du sympathique dans le glaucome. XIII Conc. Ophthalm. Hollandia, IJ, 496, 1929.

Bitrer, M.: Ueber die angeborene Defekte des vorderen Irisblattes. Klin. Mbl. Augenheilk., 6r, 277,19 I8.

BıAKe, E. M.: 'The surgical treatment of glaucoma complicating congenital aniridia. Trans. Amer. ophthal. Soc. $50,47,1952$.

Blegvad, O.: Iridoschisis. Acta Ophthal., Kbh. 29, 377, 195 I.

BlOCH, F.: Fmbryotoxon corncae posterius Axenfeld. Review of the literature and report of a case. Arch. Ophthalm. $18,68,1937$.

Bёнм, K.: Beiträge zur pathologischen Anatomie und operativen Therapie des angeborenen Hydrophthalmos. Klin. Mbl. Augenheilk., 55, 556, 19 I5.

Bojer, J.: Iridoschisis. Fssential iris atrophy. Acta ophthal., Kbh., 3I, 253, I953.

Bollack, J., Voisin, J., Camps, S.: Sur unc forme particulière de glaucome infantile: malformation de I'angle irido-cornéen, intégrité fonctionnelle. Bull. Soc. Ophtal., Paris, p. 127, $193^{8}$.

Bondr, M.: Megalophtalmus und Hydrophthalmus in einer Familie, Klin, therap. Wschr., 14, $378,1903$.

Bösirar, E.: Der Feinbau der Iris bei verschiedener Pupillenweite. Anthrop. Anz., 20, I956.

Broendstrif, J. M.: Posterior embryotoxon in three generations. Acta ophthal., Kbh. $26,495,1948$.

Broman, I.: Normale und abnorme Entwicklung des Menschen. Wiesbaden igr i.

Brons, H.: Ueber dic Vererbung des Hydrophthalmus congenitus. Inaug. Dissertation, Tubingen, 27 pp. I 937.

Brues, A.: A genctic analysis of human eye color. Am. J. Phys. Anthrop., 4, I946.

Bürstrnainder, O.: Beitrag zur Kenntnis der Missbildungen des Auges (Atypische Iriskolobome). Klin. Mbl. Augenheilk, 40, 4I5, 1902 .

Busacca, A.: Eléments de Gonioscopic. Edit. Rossolillo, São Paulo, I 945.

- Examen gonioscopique d'un cas d'hydrophtalmie. Ann. Oculist., Paris, I8I, 627, 1948.

Busacca A., Canavesi G.: Osservazione su di un caso di coloboma irideo bilaterale in gemelli monocori. Boll. Oculist. 4, 556, r952.

- Pinticart, W.: Etude gonioscopique d'un cas d'embryotoxon corneae posterius. Ophthalmologica, Basel, $I I_{5}, 28_{3}, 194 \%$.

Caballiko dei. Castillo: Iridodiastasis. Arch. Oftal. hisp. - amer., 8, 897, i948.

Carannes: Buphtalmie, aniridie. Soc. Ophtal. Bordeaux, dic. 1895, cit. da Lagrange.

Cadenas Ugidos, C.: Aniridia congenita. Arch. Oftal. hisp. -- amer., 14, 923, 1954.

Calioum, F. P.: Disease of the uveal tract. Arch. Ophthalm., 5I, 376, 1954.

Callahan, A.: Multiples abnormalities of the iris (mesodermal tissue, atrophy and holes). With secondary glaucoma and deafness (Histologic examination of one eye). Amer. J. Ophthalm., 4I, 745, 1956.

Carlevaro, G.: Contriluto alla patogenesi del glaucoma giovanile. Boll. Oculist., 20, 350, I94I.

Carter, L. F.: Progressive essential atrophy of the iris. Case history with pathologic report. Amer. J. Ophthalm., $35,522,195^{2}$.

- Progressive scnile fibrillar atrophy of the iris stroma. Amer. J. Ophthalm., 36, 967, 1953.

Cassuto, N.: Su di una malformazione iridea bilaterale. Boll. Oculist., 17,576 , $193^{8}$.

Castelli: Contributo alla conoscenza dell'anatomia patologica e all'etiologia dell'idroftalmo congenito. Ann. Ottalm., 68, p. 801 , 1940.

Ceccietro, E.: Dell'ectropion uveae congenitum e dei flocculi pupillari nell'uomo. Arch. Ottalm., I6, 286 , I908-1 909 .

Chanderer, P. A.: Atrophy of the stroma of the iris. Endothelial distrophy. Corneal edema and glaucoma. Amer. J. Ophthalm., 4I, 607, $195^{6}$.

Chang, L. W., Ojers, G. W.: Essential progressive atrophy of the iris. Amer. J. Ophthalm., 32, 368 , I949.

Charamis, J.: Acquired atrophic colobomata of the iris. Bull. Soc. héllen. Ophtal., 23, 52, I955.

CiHI, M. S.: Holes in Iris. Chinese J. Ophth., 1, 264, $195 \mathrm{I}$.

Chin, H. Y.: Primary Atrophy of the Iris. New Med. China, I, 346, I950.

Crrronis, P.: Ein Fall von Irisatrophic mit Iückenbildung nach Atropin - Glaucoma. Klin. Mbl. Augenheilk., $73,480,1924$. 
Ciotola, G.: Atrofia bilaterale congenita del foglietto anteriore dell'iride e glaucoma secondario. Boll. Oculist., 19, I015, I940.

- Ancora sull'atrofia essenziale dell'iride associata a glaucoma. Atti Soc. Oftal. ital., 13, 137, 1952.

C.lausen: Aniridia congenita und Heredität. Klin. Mbl. Augenheilk., 67, I16, I921.

Collins: Aniridia and glaucoma. Ophthal. Rev., ıo, ror, I89ı.

Cornalba, G.: Ulteriori considerazioni su tre casi di atrofia bilaterale del foglietto anteriore dell'iride e glaucoma secondario. Boll. Soc. med.-chir. Pavia, 68, 1043, 1954.

Cosmettatos, G. F.: Ueber eine Form von Pseudocolobom der Iris oder Ectropium Uveae congenitum. Arch. Augenheilk., 53, 180, 1905 .

Cresseri A.-Becherle.: Ricerche sull'ereditarietà dell'idroftalmo in paese della Valsassina. Istituto Lombardo di Scienze e Lettere. Rendiconti Cl. di Scienze, 78, p. 472. I944-1 945.

Czukrasz, I.: Beitrag zur Frage der atyphischen Iriskolobome. Ophthalmologica, Bascl 122 , 53, I95 I.

Daily, L., DaILY, R. K.: Iridectomy for early essential atrophy of the iris. Amer. J. Ophthalm., 44, 487, I957.

Dalsgard-Nielsen, E.: Buphthalmia. A survey of buphthalmic patients admitted to the eye clinic of the Rigshospital in the period of I9I0-43. Acta Ophth., 23, 49, 1945.

De Benedett, A.: Irideremia totale congenita. Ectopia lentis congenita con lussazione spontanea del cristallino e glaucoma consecutivo. Ann. Ottalm., $15,184,399$, I 886.

De BrRardinis, E.: Una famiglia con aniridia congenita. Contributo all'ereditarietà dell'irideremia. Arch. Ottalm., 57, 153, 1953 .

De Conciliss, U.: Atrofia essenziale e progressiva dell'iride. G. ital. oftal., 7, 456, 1946.

De Ferrari, G.: Su di un caso di atrofia essenziale o primaria dell'iride associata a glaucoma trattato chirurgicamente con esito fortunato. Boll. Oculist., 33, 290, I954.

Delmarcelle, Y.: Considerations sur l'hérédité du glaucome infantile. Journ. Genet. Humainc 6 , 33, I 957 .

De Schweinizz, G. E.: The clinical features and etiologic factors of essential progressive atrophy of the iris and the formation of holes in its tissue. Trans. Amer. ophthal., Soc. 24, 122, 1926.

- Essential progressive atrophy of the iris; a second communication. Arch. Ophthalm., 56, io, 1927.

Dickinson, F.: A case of congenital aniridia. Trans. int. ophthal. Congr., 4IO, I95 I.

Dorne, P. G.: Congenital malformation of iris. Proc. R. Soc. Med. 15 (Ophthal.), 7, 1922.

Dudinow, O. A.: Ein seltener Fall von Membrana pupillaris persistens corneae adhacrens. Z. Augenheilk., $81,33^{6}, 1933$.

Duggan, J. N., Nanavati, B. P.: A family with aniridia. Brit. J. Ophthal. 1 I, 447, 1927.

Duke-Elder, S.: Text-Book of Ophthalmology, Ed. U. Kimpton 1954, vol. $5^{\circ}$.

Duncan, W. J. L.: Personal communications 1937 cit. da Anderson.

Dupuy-Detemps: Sur une forme spéciale d'atrophie de l'iris au cours du tabes et de la paralysie générale. Ses rapports avec l'irregularité et les troubles réflexes de la pupille. Ann. Oculist., Paris, I34, 190, I905.

Eissler, P.: Die menschliche Iris. In: Schieck, F. und Brückner, A., Kurzes Handbuch der Ophthalmologie, Berlin, 1930.

EL Balky, M. A.: A peculiar case of polycoria. Bull. Soc. Ophtal. Egypte, 29, I53, 1936.

Elter: Trans. Amer. ophthal. Soc. 26, 306, 1928. Citato da Crotola.

Eskelund, V.: Structural variations of the human iris and their heredity. Copenhagen, I938.

Esteban, A., Wolf, J. A.: Arch. Oftal B. Ayres 23, 141, 1948. Gitato da Streiff.

FALLS, A. F.: A gene producing various Defects of the Anterior Segment of the Eyc. With a Pedigree of a Family. Amer. J. Ophthalm., 32, 41, 1949.

FArina, F.: Sopra un caso di contemporanea presenza d'iridemia parziale in un occhio e corcctopia nell'altro, con idroftalmia congenita bilaterale. G. Oculist. 4, 14 I e i $50,1923$.

Feingord, M.: Essential atrophy of the iris. Amer. J. Ophthalm., I (N.S.), I, 19 I8.

Felix, C. H.: Kongenitale familiäre Cornea plana. Klin. Mbl. Augenheilk., 74, 7 I0, I925.

Filippi-Gabardi, E.: Atrophie superficielle en secteur de l'iris dans le glaucome chronique irritatif. Istanbul Göz Klinigi Bülteni, 3, I 955 .

Fine, M., Barkan, H.: Essential progressive iris atrophy. Amer. J. Ophthalm., 20, 277, i937.

Françors, J.: La gonioscopie. Librairie R. Fonteyn, Louvain, 194.8. 
- The persistence of embryoic mesodermal tissue in the iridocorneal angle in cases of congenital glaucoma and other malformations. Bull. Acad. Méd. Belge, I6, 33, I95I.

- A propos d'une famille présentant des anomalies oculaires du type colobomateux depuis colobome unilatéral de l'iris jusqu'à l'anophtalmie bilatérale, associé au syndrome de Bardet-Bield. Bull. Soc. belge Ophtal., Io4, 342, 1953.

- Encyclopédie Méd. Chir. Ophtalmologie 21225 F oo, pag. 4, Ed. E.T., Paris, 1956.

- De Rouck, A.: Aplasia congenita e ereditaria dell'iride. G. ital. oftal., 6, 309, 953.

Frank Kamenestzki, S. G.: Eine eigenartige hereditäre Glaukomform mit Mangel des Irisstromas und geschlechtsgebundener Vererbung. Klin. Mbl. Augenheilk., 74, 133, 1925.

Freerksen, E.: Die Struktur der menschlichen Iris und ihre Vererbung. Z. Anat. Entw.-gesch., Io9, $193^{8 .}$

Fritz, W.: Sitzungsber, Kaiserl. Akad. Wissensch. Wien II5, 3, 485, 1906. Gitato da Streiff.

GallengA, C.: Contributo allo studio dell'ectropion uveae e dell'orlo pupillare. Arch. Ottalm., I2, 4I I, I9041905 .

- Dell' "ectropion uveae congenitum" e dei cosiddetti flocculi pupillari con speciale riguardo al loro rapporto col Sinus anularis di Szict. Arch. Ottalm., I3, 132, I905-1906.

- Matteucci, R. P.: Idroftalmo. Relazione al XXXIX Congr. Soc. Oftal. ital., I952.

Garden, R. R., Wear, A. R.: Iridoschisis in a case of chronic primary glaucoma Brit. J. Ophth., 33, 509, I949.

Gasteiger, H.: Ueber Misshildungen im Bereiche der Regenbogenhaut und des Kammerwinkels und ihre klinische Bedeutung. Klin. Mbl. Augenheilk., 99, 36, 1937.

Gates, R. R.: Human Genetics. New York. I946.

Gault, E. L.: Personal communications, 1937, cit. da Anderson.

Georgiades, G.: Essential atrophy of the iris. Arch. Soc. Opht. Grece Nord, 3, 30, i 954.

Gianferrari, L., Cantoni, G.: Manuale di genetica. Vallardi, Milano, I94I.

Gianferrari L., Cresseri A., Maltarello A.: Ricerche sull'ereditarietà dell'idroftalmo e della cataratta congenita in paesi delle Prealpi Orobiche. Acta Gen. Med. Gem. 3, I, 1954 .

Gioor, A.: Klinische Beobachtung eines Falles von Koloboma iridis partiale incompletum. Arch. Augenheilk., 37, I $59, \quad 1898$.

GLüH, B.: Ueber angeborenes Fehlen des vorderen Irisblattes. Z. Augenheilk, 63, 175, 1927.

Goln, D. A.: Essential Atrophy of the iris. Bull. Pract. Ophth., 17, r8, 1947.

Gradle, H. S., Sugar, H. S.: Concerning the chamber angle. II. Exfoliation of the zonular lamella and glaucoma capsulare. Amer. J. Ophthalm., 23, 982, I940.

Graves, B.: Certain clinical features of the normal limbus. Brit. J. Ophthal., ${ }_{1} 8,3^{\circ} 5$, I 934.

GreefF: Ueber das Vorkommen von geschlitzten Pupillen beim Menschen. Arch. Augenhcilk., 74, 1, I9I3.

Greenberg, R.: Congenital bilateral coloboma of iris and choroid. Amer. J. Ophthalm., 30,63 I, 1953.

Gregg, Swan, Rones, Irvine, Smith, Guerry, Long, Danielson: Les lésions de l'angle irido-cornéal et la buphtalmie. XII Congrès des Pédiatres; Algèr, Mai 1951. J. Opht. soc., Fév. I952.

Grignolo, A.: Su due casi di aderenze congenite irido-corneali multiple. Boll. Oculist., 28,641 , I949.

Griscom, M.: Essential atrophy of the iris. Amer. J. Ophthal. Io, 647, 1927.

Gualdi, G.: Un caso di coloboma tipico della coroide in un occhio e dell'iride nell'altro. Boll. Soc. med.-chir. Modena, 5I, 97, I95I.

Guerra Grande, J. M.: Coloboma tipico, bilateral y simultaneo de iris, c. ciliar, cristalino y corioretina, a la entrada nel nervio optico. Arch. Oftal. hisp.-amer., r I, I39 r, r95 I.

Guerrero, R. A.: Un caso de coloboma simple de iris de situacion atipica y acompañado de cataracta congenita y de retinopatia macular. Rev. Policlin. Caracas, I9, 207, I951.

GürtLer, E.: Ueber das Verhalten der vorderen Grenzschicht der Iris und den Reduktionsgrad derselben beim Menschen von der Geburt bis über die Reifungsperiode. Sitzungsber. 4. Kongr. Anthrop. u. Ethnol, Wien 1952 .

Hagedoorn, A.: Congenital anomalies of the anterior segment of the eye. Arch. Ophthalm., I7, $223,1937$.

HaIk, G. M., Lyda, W., Waugh, R. L.: Iridoschisis - Report of an additional case. Arch. Ophthalm., $4^{8,}$ $40,1952$.

HARMs, C.: Einseitige spontane Lückenbildung und der Iris durch Atrophie ohne mechanische Zerrung, Klin. Mbl. Augenheilk., 4I, 522, I 903 . 
Heath, P.: Essential atrophy of the iris as histopathologic study. Trans. Amer. Ophthal. Soc., 51, I67, I953. - Essential atrophy of the iris. A histopathologic study. Amer. J. Ophthalm., 37, 2 19, 1954.

Henderson, J. W., Benedict, W. L.: Essential progressive atrophy of the iris. Report of a case. Amer. J. Ophthalm., 23, 644, 1940.

Hermann, P.: Deux cas d'aniridie totale d'origine traumatique. Bull. Soc. Ophtal. France. 474, i954.

Hescri, M.: Pigmentierungsverhältnisse der menschlichen Iris nach Alter und Geschlecht. Verh. Ges. Phys. Anthrop., 5, I93I.

Hess, G.: Ein Beitrag zur Kenntniss der nicht traumatischen Iridodialyse. Klin. Mbl. Augenheilk., 3o, ro3, I 892 .

Hu, J.: Total disappearance of the iris due to the essential atrophy. Amer. J. Ophthalm., $3^{I}$, I I68, I948.

Huber, A.: Ophthalmologische Untersuchungen an EZ und ZZ. Arch. J. Klaus-Stift., 6, I931.

Huercamp, B.: Irisschwund bei Xeroderma pigmentosum. Klin. Mbl. Augenheilk., II9, 286, I95I.

— Zwei Typen des progressiven durchgreifenden Irisschwundes. Klin. Mbl. Augenheilk., I 21, 654, I952.

Kaises, B.: Ueber Embryotoxon corneae posterius nebst einem Befund von persistierenden Resten der Membrana capsulo-pupillaris Lentis. Klin. Mbl. Augenheilk., 68, 82, 1922.

KAMEL, S.: Surgical control of glaucoma secondary to essential atrophy of the iris. Bull. Soc. Ophtal. Egypte, $46,243,1953$.

Kaminski, D.: Ein Fall von Polycorıe mit Glaukom. Russk. oftal. Zh. 7, 347, i 928 (In: Zb]. ges. Ophthal. 20, 699, I928).

Karbacher, P.: Ringförmige Zirkulärleiste des Descemetirandes. Klin. Mbl. Augenheilk., Io4, $329,1940$.

Kel.ty, R. G. G.: Traumatic aniridia - case report - Trans. Canad. Ophth. Soc. 7, 335, I 956 (In: Zbl. ges. Ophthal. 69, 96, 1956).

Kiehle, F. A., Pugmine, C.: Buphtalmos in identical twins. Arch. Ophthalm., I2, 751, 1934.

Kitahara, S.: Ein Fall von schlitzförmiger Pupille mit atypischen Iriskolobom und anderen Augenanomalien. Acta Soc. ophthalm. jap. 38, 123, 1934 (In: Zbl. ges. Ophthal. 3I, 690, 1934).

Kitte., V.: Beobachtungen bei familiär auftretender Irisatrophie mit Durcksteigerung. Klin. Mbl. Augenheilk., I29, $464, \quad 1956$.

Klutskens, J.: Le glaucome congenitale. Relation à la Soc. Belge d'Ophthalmogie. Bull. Soc. belge Ophtal., 94, 5, 1950.

Knapp, A. A.: A case of corectopia. Amer. J. Ophthalm. I3, I4I, I930.

Kraupa, E.: Ueber Leistenbildung der Descemeti nebst Bemerkungen zur Frage der Megalocornea und des Hydrophthalmus. Graefes Arch. Ophthal., Io7, 30, I92 I.

Kreiker, A.: Ueber genuine Irisatrophie. Klin. Mbl. Augenheilk. 76, 585, 1926.

Kurtzmann, J. L.: Embryotoxon with associated haemangioma of the globe. Brit. J. Ophthal. $4^{I}, 590$, I 957 .

Jaenscir, P. A.: Anatomische und klinische Untersuchungen zur Pathologie und Therapie des Hydrophtalmus congenitus. Graefes Arch. Ophthal., II8, 21 , I927.

Jeancon, E.: Progressive essential atrophy of the iris. Amer. J. Ophthalm., I6, 3r 3, I 933.

Lagrange, E.: Traitement du glaucome infantile. Rapport à la Société Française d'Ophtalmologie, Bult. Soc. franç. Ophtal., 38, 5, 1925 .

IANe, L. A.: Primary progressive atrophy of the iris. Ophthal. Rec., 26, 285, I9I 7 .

Larmande, A. M.: L'atrophie progressive et essentielle de l'iris. Arch. Ophtalm., Paris 13, $586,1953$.

- Arroyo, Y.: Atrophie essentielle de l'iris et troubles endocriniens. Bull. Soc. Ophtal. France, p. 314 , I953.

LAsky, A. M.: Essential progressive atrophy of the iris. Amer. J. Ophthalm., 3I, I I31, I948.

Leland, F., Carter, M. D.: Progressive essential atrophy of the Iris (Case history with pathologic report). Amer. J. Ophthalm., 35, 522, 1952.

Levy, W. J.: Congenital iris lesion. Brit. J. Ophthal., 4I, 120, 1957.

Licsko, A.: Durch Irisatrophie hervorgerufenes Glaukom. Klin. Mbl. Augenheilk., 71,456 , 1923.

-- Vererbte Irisatrophie. Klin. Mbl. Augenheilk., 78, I927.

Liebiтzкy, H.: Zur Kenntnis der angeborenen Irisanomalien. Aplasia semicircularis iridis circumpupillaris. Arch. Augenheilk., IOI, 771, I929.

Lijo Pavia, J.: Iridoschisis - Una nueva observacion. Arch. Oftalm., B. Aires. 26, 43I, I95I.

Lindarkg, J. G.: Beitrag zur Kenntnis der congenitalen sog. Aniridie. Fälle totaler und partieller Aniridie 
und ein "Aniridie" - Fall mit beibehaltener Iris in einer und derselben Familie. Klin. Mbl. Augenheilk., $70, \mathbf{1} 33,1923$.

Linn, I. C., Linn, J. G. Jr.: Detachment of the Anterior Layer of the Iris (Iridoschisis). Amer. J. Ophthalm., 32, I 700,1949 .

LodA TO, G.: Le altcrazioni trofiche dell'iride nella tabe dorsale. Boll. Oculist., 3, 869, 1924.

-. Su di un caso di disinserzione spontanea dell'iride con notevole alterazione dello stroma irideo. G. ital. oftal., 4. $120,195 \mathrm{I}$.

Lönlein, H.: Zur Kenntnis der essentiellen fortschreitenden Irisatrophie mit Lochbildung und Glaukom. Klin. Mbl. Augenheilk., II 8, 379, I95I.

Loewenstein, A., Foster, J., Slenge, S. K.: A further case of Iridoschisis. Brit. J. Ophthal., 32 , I29, 1948.

Magistretti, A.: Ricerche genetiche sull'atrofia essenziale dell'iride. Atti del XLII cong. Soc. Oftal. ital. I956.

Magnussen, K.: 7.ur Bestimmung der Irisstruktur in menschlichem Auge. Erbartz I I, I943.

Mandras, G.: Complete bilateral coloboma of the iris, the lens, the choroid and the optic nerve. Bull. Soc. hellén. Ophtal. 22, 233, 1954.

Mans, I.: On congenital hyaline membranes on the posterior surface of the cornea. Brit. J. Ophthal., I7, 449, 1933.

- Developmental abnormalities of the eye. II Ed., Brit. Med. Ass., London, 1957.

Marin Amat, M.: Isa aniridia congenita. Soc. Med. Esp., 28, 667, 269, 1954.

Massimeo, A.: Idroftalmo monolaterale in una coppia di gemelli MZ. Boll. Oculist., 35, 824, 1956.

Matteucat, P., Vannini A.: Il tessuto mesodermico dell'angolo irido-corneale nell'idroftalmo. Atti Soc. Oftal. Lombarda, 7, 44, 1952 .

Max, W.: Irisatrophie und epibulbäres Karzinom bei Xeroderma pigmentosum Klin. Mbl. Augenheilk. I3, 75 , 1912 .

Mc Culzoch, C.: Iridoschisis as a cause of Glaucoma. Amer. J. Ophthalm., 33, r398, 1950.

Mc Keown, H. S.: Essential progressive atrophy of the iris. Report of a case. Arch. Ophthalm., I8, 347, 1937.

Moffat, P.: Iridoschisis. Proc. Roy. Soc. Med., 43, Io i , I950.

Mornx, P. V., Gombí-Jom, D.: Curieuse lésion de l'iris d'étiologie indeterminée (Atrophie essentielle et progressive de l'iris). Bull. Soc. Ophtal. France, p. 588 , 1955.

Munihead, W. M.: Atrophy of the iris. Trans. ophthal. Soc. U. K., 56, 266, 1936.

Paez Allende, F. S.: Atrofia essencial del iris en una paciente con tubercolosis pulmonar. Sem. med.; B. Aires, IOT, 650, I952.

Pagliarani, N.: "Status dysraphicus" ed atrofia essenziale progressiva dell'iride. Riv. oto-neuromoftalm., $28,46 \mathrm{I}, \mathrm{I} 953$.

PANTER, E. G.: Two cases of iridoschisis. Amer. J. Ophthalm. 34, 297, I95 I.

PAC, H.: Hornhaut-dystrophie und essentielle fortschreitende Irisatrophie mit Lochbildung und Glaukom. Klin. Mbl. Augcnheilk., I22, 732, I953.

Paufigue, L., Erienne, R., Bonnet, J. L.: L'embryotoxon postérieur de la cornée. Ann. Oculist., Paris, $18_{3}$, $8 \mathrm{I}, 195^{\circ}$.

Paulo Filio, A.: Atrofia da iris. Rev. bras. oftal., IO, 229, $195^{2}$.

Pellaty, A.: Hypoplasia familiaris des vorderen Irisblattes, mit konsekutiver Mydriasis. Klin. Mbl. Augenheilk, 77,368, I 926 .

Peters, A.: Das Glaukom. Graefe-Saemisch., Hdb. ges. Augenheilk. 3 Aufl, Berlin, Springer I 930.

Plas, E.: A contribution to the Aetiology of Essential Progressive Atrophy of the Iris. Csl. Oftalm. 6, 29 I, I950.

Poliakowa-Spector, M. I.: Zur Frage der beidseitigen, symmetrischen primären Atrophie des vorderen Irisblattes. Vestn. Oftalm. 1 I, 371, 1937. (In: Zbl. ges. Ophthal., 40, 615, 1938) .

Polte: Mchrcre Fälle angeborener Irismissbildung. Arch. Augenheilk., 48, 75, I903.

Post, M. H.: Essential progressive atrophy of the iris. Amer. J. Ophthalm., 22, 755, 1939.

Potts, R. C.: Hydrophalmia in identical twins Trans. Amer. ophthal. Soc., 256 , I950.

Purvis, V. B.: Bilateral buphthalmos with congenital anomalies of iris and subluxated lenses. Amer. J. Ophthalm., 27, 1433, 1944 .

Reed, FAlts: Albero genealogico con discussione del mosaicismo germinale nell'uomo. Amer. J. Human. Genet., $7,28, \mathbf{1} 955$. 
Rrmky, E.: Embryotoxon corneae posterius (Axenfeld). Klin. Mbl. Augenheilk, 78, 5i2, ig27.

Rieger, H.: Beiträge zur Kenntnis seltener Missbildungen der Iris. I. Membrana iridopupillaris persistens. Graefes Arch. Ophthal., I3I, 523, 1934.

- II. Ueber Hypoplasie des Irisvorderblattes mit Verlagerung und Entrundung der Pupille. Graefes. Arch. Ophthal., 133, 502, 1935 .

Rochat, G. F., Mulder, W.: On progressive atrophy of the iris with formation of holes and glaucoma, Brit. J. Ophthal., 8, $3^{62}$, I 924 .

Rones, B.: Essential atrophy of the iris with pathological report. Amer. J. Ophthalm. 23, I63, 194.0.

Rossano, A.: Absence presque complète du feuillet mésodermique de l'iris dans deux générations. Hypertension concomitante et polyurie dans un cas. Arch. Ophtalm., Paris $5 I, 550,1934$.

Rossfitr, D.: Dysgenesia mesodermalis e vizi di cuore. Ann. Ottalm. 78, 41 7, 1952.

- Betetro, G.: Glaucoma familiare e quadri malformativi multipli. Ann. Ottalm., 82, $139,1956$.

Rüвel, E.: Angeborene Hypoplasie bzw. Aplasie des Irisvorderblattes. Klin. Mbl. Augenheilk., 5I, I74, 1913.

Ruby F. Mak: Essential atrophy of the iris. Amer. J. Ophthal., 27, I 7 I, 1944.

SABA, V.: Rara malformazione del limbo corneale in soggetto idrocefalico. Ann. Ottalm., 55, 446, 1927.

SAKIC, D.: Un cas de dysgenesis mesodermalis corneae et iridis. Ophthalmologica, Basel. 123, 31, 1952.

Santonastaso, A.: Sull'etiopatogenesi dell'idroftalmo. Contributo clinico e istopatologico. Ann. Ottalm., 64, 405 e $437,1936$.

SANTORI, F.: Su di una speciosa forma di atrofia dell'iride in subluxatio lentis. Boll. Oculist., 8, 31, 1929.

SCHADE, H.: Vaterschaftsbegutachtung, Stuttgart. 1954. Zur Untersuchung der Irisstruktur für die Vaterschaftsbegutachtung. Homo, 6, I955.

Scharf J.: Zur fortschreitenden Irisatrophie mit Lochbildung und Drucksteigerung (Versuch ihrer Deutung aus der gonioskopischen Beobachtung zweier Fälte) Klin. Mbl. Augenh. Io6, 41 I, 1954 •

Schnyder, U. W., Landolt, Martz, G.: Syndrome de Klippel - Trennany avec colobome irien atypique. Génét. Humaine, 5, I, 1956 .

Schwaegerle, F.: Irisstruktur und Augenfarbe bei EZ und ZZ. Z. Menschl. Vererb.-u. Konst.-lehre 22, $193^{8 .}$

Seefelder, R.: Das Verhalten der Kammerbucht und ihres Gerüstwerkes bis zur Geburt. Graefe-Sacmisch, Hdb. ges. Augenheilk. 2. Aufl,, I. Bd., I. Abtl. Anhang zu Kap., II, 34, I9ı.

- Wolfrum: Zur Entwicklung der vorderen Kammer und des Kammerwinkels beim Menschen, nebst Bcmerkungen über ihre Entstehung bei Tieren. Graefes Arch. Ophthal., 63, 430;, 1906.

Sivasubramaniam, P., Hoole, T.: Bilateral essential atrophy of the iris. Brit. J. Ophthal., 39, I I9, 1955.

Sоммев, G.: Ueber die zum Formenkreis der angeborenen Aniridie gehörigen Hornhautveränderungen. Klin. Mbl. Augenheilk., 128, 459, 1956.

Sorsiy, A.: Genetics in Ophthalmology. Butterworth, London, 195I.

Speciale-Giringione, F.: Contributo all'anatomia e patogenesi della cosiddetta aniridia (Vizio di sviluppo dell'iride) Clin. oculist., Palermo I5 $_{5}$ I8, I9 5 .

Streiff, J.: Ueber eine untere Irismulde und über Iristypen und Uebergänge zu Anomalien. Klin. Mbl. Augenheilk., 54, r9г5.

Streiff, E. B.: Dysplasie marginale postérieure de la cornée dans le cadre des manifestations irido-cornéennes. Ophthalmologica, Basel, is 8, 8i 5 , 1949.

Sugar, H. S.: Concerning the chamber angle. I. Gonioscopy. Amer. J. Ophthalm., 23, 853, 1940.

Sverdlick, J., Irirbarren, F.: Atrofia essencial progresiva del iris bilateral. Arch. Oftal. B. Ayres, 26,2 i , 1951.

Takagr, A., OdA, K.: Ein Stammbaum von Buphthalmus. Acta Soc. Opht. Jap., 37, 1765-1170, 1934 (In: Zbl. ges. Ophth., 3I, 33, I934).

Tavolara, L.: Il quadro gonioscopico dell'embriotoxon corneae posterius. Boll. Oculist., 28, 9 I, I949.

Theodore, F. H.: Arch. Ophthalm., 31, 138, i944. Git da Streiff.

Uerrero, R. A.: Un caso de coloboma simple del iris, de situacion atipica y acompanado de cataracta congenita y de retinopatia macular. Rev. Policlin. Caracas, 19, 207, I951.

Unger, L.: Beitrag zur sogen. Dysgenesis mesodermalis corneae und iridis (Rieger). Ophthalmologica, Basel, $132,27,1956$.

Uribe-Troncoso, M., Castroviejo, R.: Microanatomy of the eye with the slitlamp microscope. Amer. J. Ophthalm., $19,481,1936$. 
Valentin-Gamazo, I.: Coloboma tipico congénito monocular del iris, retina y nervio optico. Arch. Oftal. hisp.- amer., $17,165,1957$.

VAN BEuningen, E. G. A.: Vergleichende gonioskopische Untersuchungen fortschreitender, nichtdurchgreifender Irislochbildungen Klin. Mbl. Augenheilk., II 9, 279, I951.

Van Boraert, L.: Les dysplasies neuro-ectodermiques. Rev. neurol., 63, 353, 1935.

VAN Duyse: Contribution à l'étude des membranes pupillaires persistentes. Ann. Oculist., Paris, 9.5, I3, I886.

VANninI, A.: La gonioscopia nel glaucoma primitivo. Rass. Ital. Ottal., $21,65,1952$.

- La gonioscopia nell'idroftalmo. Rass. It. Ottal., $195^{2}$.

VeDeneEva: Atrofia essenziale dell'iride con glaucoma. Vestn., Oftalm. 5, 36, 1956.

VEGA DE LA E.: Fortschreitende Atrophie der nicht entzündeten Iris nach Chozioretinitis syphilitica. Klin. Mbl. Augenh. 7I, 44, 1923 .

VeIRs, E. R.: Iridoschisis. Amer. J. Ophthalm., 32, 261, 1949.

Velhagen, K. jr.: Doppeltes Gerontoxon. Klin. Mbl. Augenheilk., 85, 264, 1930.

Virkow, H.: Mikroskopische Anatomie der aüsseren Augenhaut u.s.w. Graeie-Saemisch, Hdb. ges. Augenheilk. 2. Aufll., 1. Abtl., 282, 1910.

Vogt, A.: Handb. Erbbiologie, 3, 67r, 1940.

WaARdenburg, P. J.: Erbartz., 7 , 1934 .

- Das menschliche Auge und seine Erbanlagen. Im Haag. 1932.

- Verschiedenhciten der Irisstruktur in der niederländischen Provinz Gelderland. Actes IV Congrès intern. Anthr. \& Ethnol. Vienne, 1952.

- Struktur der menschlichen Iris. Vorschlag einer übersichtlichen Typeneinteilung. Z. Morph. Anthrop., $4^{6,}, 1954$.

- Variabilität und Erblichkeit der Struktur der menschlichen Iris. Homo, 5, 9954.

- Ein autosomal-dominantes Strukturmerkmal der menschlichen Iris. Acta Genet. Med. Gem., 4, I955.

- Mündliche Mitteilungen.

WaITE: Essential Atrophy of the iris. Davis birthday vol. 1937.

Wallenberg: Dtsch. med. Wschr., 3i, 1275 , I907.

- Erbliches, juveniles Glauckom in vier Generationen Kasuistische Mitteilungen. Berich über die Verhandlung der Abteilung für Augenh., r9ro, rec. Klin. Mbl. f. Augenheilk., 48, 495, 1910.

WeEkers, S. e R.: Contribution à la pathogénie du buphtalmos. Bull. Soc. Belge d'Ophtal. 94, 258, 1950.

Weniveer, J.: Irisstruktur und Vererbung. Z. Morph. Anthrop. 34, 1934.

- Die anthropologischen Methoden der menschlichen Erbforschung. In Just: Handb. Erbbiologie, 2, 1940.

- Variabilität der Struktur der menschlichen Iris. Homo, 5, I954.

Westerlund, E.: On the heredity of congenital hydrophtalmos. Acta ophthal., Kbh.. p. 330, 1943 .

- Clinical and genetic studies on the primary glaucoma diseases. Opera ex domo biologiae. Vol. 12, Copenhagen, E. Munksgaard, 1957.

Wighmann, D.: Ueber die Vererbung der Iriskrypten und ihren Zusammenhang mit der Irispigmentierung. Acta Gen. Med. Gem., 2, 1953 .

Winkelman: Essential iridal atrophy and glaucoma. Ophthalmologica, Basel, $x_{32}^{2}, 34^{2}, 195^{6}$.

Wölfflin, E.: Klinischer Beitrag zur Kenntnis der Struktur der Iris. Arch. Augenheilk., 45, 1902.

ZANEn J.: Atrophie progressive essentielle de l'iris et de la choroide. Bull. Soc. belge Ophtal., 93, 420, 1920.

Zentmayer, W.: Essential atrophy of the iris. Amer. J. Ophthalm., I, 5 ro, igi8. 


\section{RÉSUMÉ}

Les AA. rapportent le cas d'un couple gémellaire dizygote de sexe différent, âgé de 7 mois. Les deux jumeaux étaient atteints d'atrophie de l'iris, compliquée chez le jumeau masculin de buphtalmie acquise environ au 6 mois.

Les AA. avancent l'hypothèse que l'hérédité primaire consiste dans l'atrophie irienne et que dans ce cas la buphtalmie est secondaire à cette malformation.
SUMMARY

The Authors report on dizygotic twins of 7 months and of different sex. Both twins were affected by iris atrophy, which in the male was associated with buphthalmos acquired at about 6 months of age.

The hypothesis is made that the primitive heredity consists in the iris malformation and that in this case the buphthalmos is secondary to it.

\section{ZUSAMMENFASSUNG}

Es wird über ein 7 Monate altes dizygotisches Zwillingspaar verschiedenen Geschlechtes berichtet. Beide Zwillingen wiesen eine Irisatrophie auf, die bei dem männlichen Zwilling durch einen ungefähr um den 6 Monat erworbenen Buphthalmus ompliziert war.

Es wird angenomen, dass die primäre Vererbung in der Irismissbildung bestehe und dass der Buphthalmus in diesem Falle dieser Missbildung sekundär sei. 